\title{
Syntaxe des proverbes binaires: coordinations et parataxes
}

\section{Syntax of Binary Proverbs: Coordinations and Parataxes}

Alexandra Oddo

RECIBIDO: 22 DE FEBRERO DE 2017 ACEPTADO: 13 DE JUNIO DE 2017

Département d'espagnol

Université de Paris Nanterre

200 Avenue de la République. Nanterre, 92000. Francia

alexandra.oddo@parisnanterre.fr

Orcid ID: 0000-0003-3519-9593

Resumen: En los años noventa, los estudios sobre la estructura binaria de los refranes fomentaron nuevos enfoques de estudio para la definición de la dimensión sintáctica y semántica de los enunciados sentenciosos. El esquema funcional semántico que asocia las distintas partes del refrán mediante un nexo de tipo implicativo fue analizado por Riegel, y más tarde Anscombre y Kleiber, y permite entender cómo se articulan estos enunciados que, por sus múltiples facetas y su peculiar funcionamiento, a menudo han sido apartados de las gramáticas y de los estudios lingüísticos. Dicha implicación que une a los dos miembros de un refrán binario se presenta en efecto en la gran mayoría de los enunciados sentenciosos. Cuando es así, el vínculo sintáctico que se establece entre los miembros del refrán puede ser descrito gracias a la impli- cación en el ámbito semántico y gracias a la parataxis o al asíndeton en el ámbito sintáctico, ya que ningún elemento formal declara la relación que liga a los dos miembros del refrán. Sin embargo, la observación del grupo heterogéneo de los refranes permite cuestionar la validez de dicha propiedad lingüística para el conjunto del refranero. Con este objetivo, el presente estudio pretende encontrar y analizar otros modelos de articulación, como pueden serlo la coordinación copulativa y adversativa, porque dichas estructuras sintácticas a todas luces pueden alterar el contenido semántico de conjunto del enunciado.

Palabras clave: Paremiología. Sintaxis. Implicación. Coordinación. Semántica. 


\begin{abstract}
Works on binary proverbs have constantly brought new thoughts to the involved researchers in order to define the sentencious postulate in its syntactic and semantic dimensions. The assumption of a semantic pattern of operation based on the involvement of the terms of the postulate developed first by Riegel, and then by Anscombre and Kleiber (as of 1994) establishes proverbial postulate. The implication, which connects the two parties of a binary proverb is certainly present in the vast majority of proverbs. In this case, the syntactical parties of the statement can be described through the semantic involvement and through parataxis or asyndeton syntactically because no
\end{abstract}

Résumé: Les travaux sur la binarité des proverbes n'ont cessé d'apporter de nouvelles pistes de réflexion aux parémiologues soucieux de définir l'énoncé sentencieux dans ses dimensions syntaxique et sémantique. Le postulat d'un schéma sémantique de fonctionnement fondé sur l'implication des termes de l'énoncé développé par Riegel puis par Anscombre et Kleiber (dès 1994) permet de mieux saisir l'énoncé proverbial, dont les manifestations multiples sont parfois déroutantes sur le plan linguistique, notamment en raison de la complexité des liens qui unissent les différents éléments qui s'agencent dans tout proverbe. Le "pivot implicatif", qui met en relation les deux membres d'un proverbe binaire est en effet présent dans une très grande majorité d'énoncés sentencieux. Dans ce cas, l'enchaînement syntaxique des membres de l'énoncé peut être décrit grâce à l'implication sur le formal element exists to specify the type of relationship established between both parties. In comparison to this system, however, some proverbs question the validity of this property for the overall majority of the proverbs. The objective of this research is to analyze the presence of other models (including case and copulative adversative coordination) because the syntactic structure can affect the semantic domain in the sense that the relationship between parties of the proverb may be significantly different.

Keywords: Paremiology. Syntax. Involvement. Coordination. Semantics.

plan sémantique et grâce à la parataxe ou à l'asyndète sur le plan syntaxique, nul élément formel ne venant préciser le type de relation qui s'instaure entre les deux parties. En marge de ce système, pourtant, un certain nombre de proverbes remettent en question la validité de cette propriété pour l'ensemble des énoncés sentencieux. L'objectif de la présente étude est d'analyser la présence d'autres modèles de fonctionnement, notamment les cas de coordination copulative et adversative, dont la structure syntaxique de surface peut avoir une incidence dans le domaine sémantique en ce sens que la relation existant entre les deux membres du proverbe peut y être sensiblement différente.

Mots clés: Parémiologie. Syntaxe. Implication. Coordination. Sémantique.

es travaux sur la binarité des proverbes ont constitué l'une des bases fondamentales de la définition de l'énoncé sentencieux, notamment dans sa dimension syntaxique et sémantique. Ces études ont mis en évidence, au sein des proverbes dits binaires, la présence d'un schéma sémantique de fonctionnement fondé sur l'implication entre les différents termes de l'énoncé. Observé et analysé à partir des travaux de Riegel puis de Kleiber (1989) et Anscombre (1994; 1997) dès la fin des années quatre-vingts, ce "pivot implicatif", tel qu'il est nommé dans les premiers articles, est un schéma de fonctionnement qui, en dépit des manifestations linguistiques multiples -et parfois déroutantes- de l'énoncé sentencieux, permet de caractériser, entre autres propriétés, un énoncé comme appartenant à la classe des proverbes. Dès lors, 
ni la présence de différentes "matrices ou moules proverbiaux" (Gómez-Jordana 2012a; 2012b; Anscombre 2012a; 2012b), ni le nombre de parties présentes dans un proverbe n'entame la validité de l'enchaînement logique qui rend interdépendants sur le plan sémantique les deux éléments de l'énoncé.

Il convient d'ajouter que ce lien sémantique se matérialise le plus souvent précisément en l'absence d'un lien coordonnant les propositions associées, et ce dans de nombreuses langues, dont Anscombre avait dressé un rapide bilan en 2012: "Par ailleurs, les proverbes ont souvent recours à des relations paratactiques: Hoy por ti, mañana por mí (esp.); Spare the rod, spoil the child (ang.); Tel qui rit vendredi, dimanche pleurera (fr.); Etxean ikusia, umeak ikasia (basque); Wie der Vater, so der Sobn (all.); etc." (2012a, 22-23).

Le fonctionnement du proverbe peut donc être schématisé sur le plan sémantique et syntaxique avec, d'une part, l'enchaînement des éléments du proverbe qui peut être décrit grâce à l'implication sur le plan sémantique; et d'autre part, la matérialisation de cette relation par le biais de la parataxe ou de l'asyndète, deux procédés où nul élément formel ne vient préciser le type de relation qui relie les deux membres de cette structure binaire.

En marge de ce système, pourtant, un certain nombre de proverbes remettent en question la validité de cette propriété pour l'ensemble des énoncés sentencieux. L'objectif de la présente étude est d'analyser d'autres modèles de fonctionnement. Et notamment des cas de coordination marqués par la présence de conjonctions dont le sémantisme peut avoir une incidence sur la lecture de la relation qui existe entre les deux propositions dans le domaine sémantique. Pour en vérifier, par la suite, la validité en diachronie grâce à une typologie des différentes évolutions auxquelles ces conjonctions vont être soumises.

\section{TERMINOLOGIE ET CONCEPTS}

Notre étude prend appui sur une série de concepts fondamentaux sur lesquels il faut revenir rapidement. Les définitions les plus récentes concernant la catégorie linguistique "proverbe" font ainsi état d'un énoncé autonome et minimal pourvu de propriétés morphologiques, syntaxiques et sémantiques distinctives (Anscombre 2012a, 36). Pour les domaines sémantiques et syntaxiques qui nous intéressent plus particulièrement dans le cadre de cette étude, il est utile de revenir sur les notions de proverbe bipartite et de binarité, une binarité qu'il faudra d'ailleurs préciser puisque celle-ci pourra être rattachée au domaine de la syntaxe ou de la sémantique. 
Formellement, nous pouvons dresser une typologie à partir du nombre de "membres" ou "parties" présentes dans le proverbe. Cette typologie fait apparaître que sur le plan structurel, les proverbes ne sont pas forcément bipartites, certains d'entre eux ne faisant état que d'un seul membre en surface. Ces proverbes sont très répandus et ont été classés par Gómez-Jordana (2012b, 119-20) dans la catégorie des phrases canoniques: "La plus grande partie des proverbes canoniques de notre corpus comporte un groupe nominal sujet à article zéro -Petite pluie abat grand vent- ou à article défini singulier -L’habit ne fait pas le moine-. Les phrases attributives sont également abondantes -Charbonnier est maître chez soi-". Nous retenons donc comme faisant partie de la catégorie des énoncés sentencieux, sans pour autant être composés de deux "membres" apparents, les énoncés du type:

(1a) L'habit ne fait pas le moine

(1b) El mentir pide memoria

Dans le même ordre d'idées, l'énoncé sentencieux peut faire état d'un nombre de membres supérieur à deux. Certains proverbes sont ainsi composés de tercets ou de quatrains, selon la terminologie adoptée par Anscombre dans sa description des schémas rythmiques présents dans les proverbes (2012b, 15255) et peuvent être divisés en 3 ou 4 parties:

(2a) Donner / c'est donner, reprendre / c'est voler

(2b) Si da el cántaro en la piedra, / o la piedra en el cántaro, / mal para el cántaro

Cet éventail varié dans le nombre des membres du proverbe ne peut pourtant pas occulter qu'une structure s'impose d'une façon plus nette en parémiologie, celle du proverbe bipartite ou binaire, tel qu'on la retrouve dans:

(3a) Quand le chat n'est pas là, les souris dansent

(3b) Perro ladrador, poco mordedor ou sa variante Perro que mucho ladra poco muerde

Les travaux qui ont permis de matérialiser cette structure et d'expliciter le lien sémantique qui s'instaure entre les différents éléments qui s'agencent dans ce type de proverbes sont fondateurs pour la description du phénomène parémique. Ils mettent en évidence un schéma de fonctionnement fondé sur l'implication des termes de l'énoncé. Cette binarité sémantique est annoncée par les travaux de Riegel sur le "pivot implicatif", puis approfondie grâce aux travaux sur la nature conditionnelle de l'implication avec le postulat "Si X, Y". Ans- 
combre, dès les années 1980, propose quant à lui de distinguer la binarité de surface et la binarité sémantique, seule garante de l'existence d'un schème sémantique propre aux proverbes qu'il représentera dans ses travaux sous la forme de "P est argument pour / implique Q" (Anscombre 1994; 1996; 1997; 2000). L'analyse des exemples (1), (2) et (3) valide en effet la nature implicative de la relation qui prévaut dans le système des proverbes et rend inopérante une typologie fondée sur le nombre des parties présentes dans un proverbe. L'analyse sémantique de (3a) donne ainsi "l'absence du chat est un argument pour que les souris dansent" tout comme, en (1a) "l'habit n'est pas un argument qui fait le moine".

Dans son fonctionnement, cette binarité sémantique fait apparaître une relation d'implication entre les termes du proverbe, désignés respectivement comme thème et propos, du fait du rôle qu'ils assument dans l'énoncé. L'implication dont nous parlons est un cas de coordination sémantique dont la structure logique repose sur le lien qui opère entre un segment A (indépendant, constitué d'un thème et d'un propos) coordonné à un deuxième segment $\mathrm{Z}$ qui établit un nouveau propos sur A. Ce type de relation est résumé par Ducrot et Schaeffer dans le Nouveau dictionnaire encyclopédique du langage:

Coordination sémantique. À côté de la coordination syntaxique, qui unit les segments ayant même fonction syntaxique à l'intérieur d'une phrase, C. Bally a introduit une notion de coordination sémantique, qui se fonde avant tout sur les actes d'énonciation accomplis dans le discours. A et $\mathrm{Z}$ sont sémantiquement coordonnés si:

a) A est indépendant de $Z$, en ce sens qu'il fait l'objet d'un acte d'énonciation complet (il comporte donc un thème et un propos)

b) Z est présenté comme un propos dont $\mathrm{A}$ établirait le thème, comme une remarque à l'occasion de A. (Ducrot/Schaeffer 560)

La coordination, qui permet d'associer des propositions ou des éléments de même nature dans une phrase complexe ou dans une proposition indépendante respectivement, offre une large palette de nuances dans le domaine syntaxico-sémantique. Elle peut être copulative (elle relie alors deux propositions); disjonctive (elle présente un rapport d'exclusion ou d'alternative entre deux propositions); adversative (deux propositions sont mises en opposition); causale (elle indique que le fait exprimé par la seconde proposition est la cause du fait exprimé par la première) ou consécutive (elle indique que le fait exprimé par la seconde proposition est la conséquence du fait exprimé par la première). 
Différentes conjonctions auront vocation, dans nos langues romanes, à spécifier le type de relation qui devra être instauré entre les unités coordonnées. Comme le montre ce passage de l'Esbozo de una nueva gramática le nuancier est riche:

Las oraciones coordinadas del periodo pueden sucederse unas a otras por simple adición (coordinación copulativa). Cuando el hablante estima entre ellas diferencias de cualquier clase -temporales, espaciales, lógicas-, establece gradaciones que conducen a una copulación alternativa (coordinación distributiva). Estas diferencias lógicas pueden llegar hasta formular dos o más juicios contradictorios (coordinación disyuntiva). Por otro lado, los elementos simplemente copulados pueden ser todos afirmativos o todos negativos. Si esta homogeneidad lógica se altera más o menos, se producen gradaciones de matiz con las cuales se llega a expresar oposición total o parcial entre las oraciones (coordinación adversativa). Hablando en términos lógicos, diríamos que partiendo de la pura coordinación copulativa, podemos llegar a la expresión de la contradictoriedad (disyuntiva) o de la contrariedad (adversativa) como casos extremos. Como en el lenguaje no se trata del puro contenido lógico, sino de su interpretación en la forma interior con que el hablante percibe sus representaciones, los grados expresivos intermedios son muy numerosos, pero siempre se producen en una u otra de estas dos direcciones, según el esquema general que acabamos de trazar. (Esbozo 506)

Le système permet donc d'exprimer des relations très variées. Cela étant, le phénomène parémique constitue dans ce système un cas un peu particulier. Limplication correspond bien à une structure logique caractérisée par l'interdépendance et la solidarité des éléments présents dans l'énoncé, mais cette relation entre les deux membres de l'énoncé se réalise, dans nos exemples et dans une très grande majorité de cas, en l'absence de lien entre les deux membres. L'enchaînement des membres du proverbe est d'ailleurs lui-même un cas particulier de coordination: la parataxe (ou asyndète), ce qui suppose que le type de relation qui s'instaure entre les deux parties est implicite.

La parataxe est définie comme suit dans le Trésor de la langue française (s.v. parataxe): il s'agit de la "Juxtaposition de deux propositions entre lesquelles le lien de dépendance n'est qu'implicite, la courbe mélodique commune dispensant de l'usage d'un outil de coordination ou de subordination". Ce type de syntaxe est un cas particulier, et d'ailleurs, pour le décrire, Grevisse n'hésite pas à utiliser un proverbe (“Aide-toi, le ciel t'aidera"), cas exemplaire de parataxe, pour éclairer son propos: 
On parle de parataxe quand, entre deux phrases ou entre deux sous-phrases coordonnées (surtout sans conjonction: ver $\$ 262$, b), on distingue une relation logique que l'on pourrait expliciter au moyen d'une conjonction de subordination: c'est le cas du proverbe cité ci-contre; on pourrait le transposer par "Si tu t’aides, le ciel t’aidera". (Grevisse \$1109)

Voici brièvement et schématiquement exposée la théorie du fonctionnement sémantique et syntaxique des proverbes binaires. Ce lien discret qui réunit les deux parties du proverbe en l'absence d'élément de relation a d'ailleurs déjà fait l'objet d'une étude dans un recueil consacré à la parataxe (Achard-Bayle/ Schneider). Les auteurs y posent d'emblée l'équation qui s'établit entre la parataxe et l'implication, tout en soulignant la difficulté d'en appréhender le fonctionnement en dehors de toute marque formelle:

[...] si l'on considère encore que ces structures peuvent se réaliser en une suite de deux propositions (P, Q); si l'on considère enfin que cette réalisation binaire est suffisamment fréquente pour qu'on puisse parler d'un moule, on se demandera alors, dans le cas des structures parataxiques $(\mathrm{P}, \mathrm{Q})$, ce qui permet de réaliser la corrélation, à défaut de marque (d'enchâssement, de hiérarchisation) syntaxique. (Achard-Bayle/Schneider 95)

Pourtant, si la parataxe est bien la forme syntaxique la plus fréquente au sein des énoncés sentencieux, une étude minutieuse des recueils de proverbes montre cependant que toutes les formes qui y sont collectées ne présentent pas forcément deux propositions paratactiques. Elles introduisent au contraire, pour certaines, des marques formelles de coordination grâce à l'utilisation de conjonctions.

(5) La parole est d'argent et le silence est d'or

(6) La critique est aisée mais l'art est difficile

(7) No es por el huevo sino por el fuero

(8) Remienda tu sayo y pasarás el año

L'étude de ces énoncés particuliers, qui constituent en quelque sorte une exception, peut affiner la définition linguistique du proverbe. En effet, la présence de ces conjonctions de coordination au sein des énoncés sentencieux peut être le signe d'une relation sensiblement différente entre les deux membres de l'énoncé, en raison notamment du sémantisme inhérent à chaque conjonction et de l'amplitude du nuancier entrevu ci-dessus. 


\section{CORPUS DE PROVERBES BINAIRES FAISANT APPARAÎTRE UNE CONJONCTION DE COORDINATION: TYPOLOGIE, DONNÉES EN SYNCHRONIE, DONNÉES EN DIACHRONIE}

Les données consignées dans ce corpus font appel aux proverbiers espagnols et, dans une moindre mesure, aux collections françaises de la période médiévale et moderne. Les quelques exemples français sélectionnés doivent permettre de constater la présence d'un éventail varié de conjonctions nuançant les relations entre les propositions en présence. Chez Morawski, nous pouvons ainsi relever Qui m'aime et mon chien (n. $\left.{ }^{\circ} 1974\right)$; Len doit faire quant len peut, car on ne fait pas quant on veult (n. $\left.{ }^{\circ} 1458\right)$; et Il fait mal nourrir autruy enfant, car il

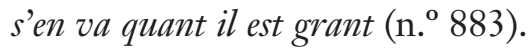

Chez Nicot (1606) aussi, quelques exemples de constructions adversatives attirent l'attention: C'est très bien dit, mais cerchez qui le face (Nicot 4); C'est grand peine que d'estre vieux: mais il ne l'est pas qui veut (Nicot 4); On sçait bien quant on s'en va: mais on ne sçait quand on reviendra (Nicot 10).

Pour finir ce bref aperçu, Le Roux de Lincy (1859) recense à la fin du XIXème siècle: Coeur de femme trompe le monde, car en luy malice abonde (Le Roux de Lincy 144); L'arc tousiours ou trop ne doibt estre tendu, car il romproit (Le Roux de Lincy 56); J'ay bon courage, mais les jambes me faillent (Le Roux de Lincy 242); F'ayme bien mes voisins, mais je n'ay cure d'eux (Le Roux de Lincy 241); La mort vient, mais on ne sçait l'heure (Le Roux de Lincy 245); Envieux meurent, mais envie ne meurt jamais (Le Roux de Lincy 221).

On retrouve ces nuances apportées par l'utilisation de conjonctions de coordination dans les collections espagnoles. Nous avons eu l'occasion d'étudier en profondeur le cas des proverbes binaires construits à l'aide d'une conjonction de coordination copulative (Oddo 2011; 2012; 2013), car ce cas est en effet particulièrement intéressant dans l'étude des proverbes tronqués: Cada uno quiere llevar el agua a su molino [y dejar en seco el de su vecino]; En todas partes cuecen habas, [y en mi casa a calderadas]; Cada loco con su tema [y cada lobo por su senda], etc., pour ne citer que les plus célèbres. ${ }^{1}$ Mais ce phénomène n'est pas systématique: d'autres proverbes se construisant à partir d'une coordination copulative (marquée par une conjonction) n'ont pas évolué vers une troncature. En voici une sélection, ils sont contre toute attente assez nombreux:

1. Les exemples fournis ici sont extraits d'un corpus diachronique de proverbes espagnols réalisé en 2013 (Oddo 2013, 209-354). 
Al villano dale el pie, y se tomará la mano

Aldeana es la gallina, y cómela el de Sevilla

Aún no ensillamos, y ya cabalgamos

Hayamos paz, y viviremos asaz

Ayúdate, y ayudarte he

Hablen cartas, y callen barbas

Cierra tu puerta y alaba a tu vecino

Cría cuervos y te sacarán los ojos

El bien suena, y el mal vuela

El mal entra a brazadas, y sale a pulgaradas

Haz bien y no mires a quién

Ládreme el perro, y no me muerda

Allégate a los buenos, y serás uno de ellos

Un padre para cien hijos, y no cien hijos para un padre

Uno muere de atafea, y otro la desea

Il faut ajouter pour ces exemples que la conjonction $y$ qui lie les deux membres de l'énoncé se conserve en diachronie, depuis le XIVème siècle, ce qui peut être considéré comme un indice de leur spécificité.

L'observation des recueils de proverbes espagnols montre aussi que d'autres conjonctions sont sollicitées, notamment celles qui permettent de réunir des propositions adversatives. Nous avons notamment des cas de construction avec mas, pero et sino.

La traición aplace, mas no el que la hace

Rogamos a Dios por santos, mas no por tantos

No donde naces, sino con quien paces

No es por el huevo, sino por el fuero

A todo hay remedio, sino a la muerte

Todo es nada, sino trigo y cebada

No te digo que te vayas, pero ahí tienes la puerta

Finalement, l'observation du corpus en diachronie permet de relever trois tendances concernant ce type d'énoncés: a) une conservation de la conjonction; b) un renforcement de la conjonction; c) un allongement de l'énoncé parémique. La syntaxe adoptée en diachronie par les proverbes suivants est mise en évidence par la forme que recensent les différents compilateurs depuis le Moyen Age (Libro de Adverbios; García de Castro; Marqués de Santillana), la pé- 
riode classique (Vallés; Núñez; Correas; Caro y Cejudo) puis la période contemporaine (Sbarbi 1872 et 1922; Doval; Junceda).

a) Conservation de la conjonction, malgré d'autres reformulations

Llegate a los buenos y serás vno de ellos (García de Castro) > Allegate a los buenos y seras vno dellos (Marqués de Santillana; Vallés); Allega te a los buenos, serás uno dellos / Llégate a los buenos y serás uno dellos (Núñez); Allégate a los buenos, i serás uno dellos / Llégate a los buenos, i serás uno dellos (Correas); Júntate con los buenos, y serás uno de ellos (Caro y Cejudo); Arrímate a los buenos, y serás uno de ellos (Sbarbi 1872); Allégate a los buenos, y serás uno de ellos (Sbarbi 1922; Doval; Junceda).

Vn padre para çient fijos, y no çient fijos para yn padre (García de Castro) > Un padre para cien hijos: y no cien hijos para vn padre (Vallés); Un padre para cien hijos y no cien hijos para un padre (Núñez); Un padre para zien hixos, i no zien hixos para un padre (Correas); Un padre para cien hijos, y no cien hijos para un padre (Caro y Cejudo; Sbarbi 1872); Un padre para cien hijos, y no cien hijos para un padre (Doval; Junceda).

Uno muere de atafea y otro la desea (Marqués de Santillana) > Uno muere de atafea: y otro la dessea (Vallés); Uno muere de atafea y otro la dessea (Núñez); Uno muere de atafea, y otro la desea / Unos mueren de atafea, y otros la desean (Correas); Uno muere de atafea y otro la desea (Sbarbi 1922); Uno muere de atafea, y otro la desea (Junceda).

Adoba tu paño, y pasaras tu año (Marqués de Santillana) > Adoba tu paño: passaras tu año (Vallés); Adoba tu paño, passarás tu año / Echa trapo, y passarás año (Núñez); Adoba paño i pasarás año; [o] Adoba tu paño i pasarás tu año (Correas); Remienda tu paño, y pasarás tu año (Sbarbi 1922); Remienda tu sayo, y pasarás el año (Junceda).

b) "Renforcement" de la conjonction

- avec un passage de Ø à $y$

Al buey por el cuerno, al onbre por la palabra (Libro de adverbios...) > $\mathrm{Al}$ buey por el cuerno, al home por la palabra (García de Castro); $\mathrm{Al}$ ombre por la palabra y al buey por el cuerno (Marqués de Santillana); Al hombre por la palabra: y al buey por el cuerno (Vallés); Al buey por el 
cuerno, y hombre por el vierbo (Núñez); Al buei por el kuerno, i al onbre por el verbo; o por la palavra (Correas); $\mathrm{Al}$ buey por el cuerno, y al hombre por la palabra (Caro y Cejudo); Al buey por el asta y al hombre por la palabra (Sbarbi 1872, 1922); Al buey, por el cuerno; y al hombre, por el verbo (Doval); Al buey, por el cuerno; y al hombre, por la palabra (Junceda).

Cría cueruo, et sacarte ha el ojo (García de Castro) > Cria cueruo, sacarte ha el ojo (Marqués de Santillana); Cria cueruo y sacarte ha el ojo (Vallés); Cría corvo y sacarte ha el ojo (Núñez); Kría el kuervo, i sakarte á el oxo (Correas); Cria cuervos, sacarte han los ojos (Caro y Cejudo); Cría cuervos y te sacarán los ojos (Sbarbi 1922; Doval; Junceda).

- passage de $y$ à mas/pero/sino

Perdió el asno los dientes, y no las mjentes (García de Castro) > Pierde el asno los dientes [y] no las mientes (Marqués de Santillana); Pierde el asno los dientes: mas no las mientes (Vallés); El lobo pierde los dientes, mas no las mientes (Núñez); Muda el lobo los dientes, y no las mientes (Caro y Cejudo; Sbarbi 1872; Doval); Pierde el lobo los dientes, mas no las mientes (Sbarbi 1922; Junceda).

Sanan las cuchilladas y no las malas palabras (Marqués de Santillana) > Sanan las cuchilladas: mas no malas palabras (Vallés); Sanan cuchilladas, mas no malas palabras (Núñez); Sanan llagas i no malas palavras (Correas); Sanan cuchilladas, mas no malas palabras (Sbarbi); Sanan cuchilladas, mas no palabras (Doval); Sanan cuchilladas, y no malas palabras (Junceda).

Justicia, y non por nuestra casa (García de Castro) > Justicia, mas no por nuestra casa (Marqués de Santillana); Justicia mas no por mi casa (Vallés); Justicia, mas no por mi casa (Núñez); ¡Xustizia! Mas no por mi kasa (Correas); Justicia, y no por mi casa (Caro y Cejudo; Sbarbi 1922); Justicia, mas no por mi casa (Doval; Junceda).

Lieua el asno la carga y non la sobre carga (García de Castro) > El asno sufre la carga: mas no la sobrecarga (Vallés); Suffre el asno la carga, mas no la sobrecarga (Núñez); El asno sufre la karga, mas no la sobrekarga (Correas); La sobrecarga mata, que no la carga (Sbarbi 1872; 1922); No mata al asno la carga, sino la sobrecarga (Doval; Junceda). 
c) Allongement sur le modèle de l'ajout d'une conjonction de coordination adversative (mas)

El buen lienzo en el arca se vende (García de Castro) > El buen paño en el arca se vende (Núñez); El buen paño en el arka se vende, mas el malo verse kiere (Correas); El buen paño en el arca se vende (Caro y Cejudo); El buen paño, en el arca se vende (Sbarbi 1922; Junceda); El mal paño en el arca se vende, mas el bueno verse quiere (Doval).

¿A do irá el buey, que non are? / ¿Do jrá el buey, que non are? (García de Castro) > A do yra el buey que no are (Marqués de Santillana; Vallés); ¿A do irá el buey que no are? (Núñez); ¿A dó irá el buei ke no are? / ¿A dónde irá el buei ke no are? -A la karnizería / ¿A dónde irá el buei ke no are, pues ke arar sabe? (Correas); Adónde irá el buey que no are? (Caro y Cejudo); ¿A dónde irá el buey que no are... sino al matadero? / ¿A dó irá el buey que no are, sino a la carnicería? (Doval; Junceda).

La pobreza non es villeza (García de Castro) > La pobreza no es vileza: mas deslustra la nobleza (Vallés); La pobreza no es vileza, mas es rramo de pikardía (Correas); La pobreza no es vileza, sino ramo de picardia (Caro y Cejudo); Pobreza no es vileza (Sbarbi); Pobreza no es vileza, mas deslustra la nobleza (Doval; Junceda).

Ce type d'analyse diachronique est essentiel pour l'observation du matériau de proverbes que nous possédons. En effet, si le proverbe "prototypique" permet, grâce à sa syntaxe, d'orienter la lecture et de poser la relation sémantique qui doit s'instaurer entre les deux membres du proverbe binaire, qu'en est-il de ces cas particuliers? S'il est vrai que le sens générique qui se dégage des proverbes qui ont constitué notre corpus, compris comme le sens littéral transcendé étudié par Kleiber -c'est-à-dire, comme il l'a proposé, un sens qui “n'est en somme qu'un hyponyme d'un sens hypéronymique qui est celui du proverbe" (Kleiber 2000, 55-56)- peut ne pas être fondamentalement altéré par cette variation, on est en droit de se demander si elle ne modifie pas, en revanche, la parasynonymie décrite au sujet des proverbes par Pottier:

Comment des discours différents peuvent-ils être considérés comme équivalents, c'est-à-dire parasynonymiques? Cela suppose qu'on se libère des mots et des structures textuelles, pour atteindre un niveau indépendant des langues naturelles. Dire: Tel père, tel fils, n'implique pas qu'on se limite à un père et à son fils. Ce qu'on retient c'est la relation entre ce 
qu'on sait du tout père par rapport à tout fils, donc à la filiation génétique, l'hérédité, la conservation probable de caractéristiques du père dans le fils. La filiation peut-être animale: (esp.): Tal el cuervo, tal su huevo; ou végétale: De doux arbres, douces pommes. En poursuivant, on atteint non pas une filiation biologique mais simplement une similitude fondée sur la relation "tout/partie": (esp.): De tal palo tal astilla. Tous ces proverbes sont équivalents dans la mesure où malgré les concrétisations spécifiques (souvent culturelles), la relation (intersection de haut niveau d'abstraction) entre les deux termes est de même nature. (Pottier 64-65)

Au regard du corpus proposé, cette assertion est-elle vraie pour tous les proverbes?

\section{LES PROVERBES BINAIRES NON PARATACTIQUES}

Ce corpus met aussi en évidence une particularité des proverbes qui les singularise encore davantage comme énoncé linguistique pourvu de propriétés morphologiques et syntaxiques propres. Cette spécificité contredit d'ailleurs une tendance naturelle de la langue, telle qu'elle a pu être décrite grâce à l'observation des connecteurs argumentatifs en diachronie. Concernant l'espagnol, Boix expliquait ainsi une tendance dans l'évolution des langues à l'effacement des connecteurs argumentatifs:

Parler ici d'effacement ne revient pas à dire que les opérations argumentatives disparaissent en tant que telles ou que le discours perdrait toute logique. Ce que l'on peut observer, c'est un phénomène de diminution sensible de la quantité présente de ces "mots du discours" chers à Oswald Ducrot: la mesure comparative diachronique montre que les connecteurs, qui viennent spécifier les opérations logiques à effectuer sur les constituants de l'énoncé, ont tendance à se raréfier [...] Non pas, répétons-le, que ces derniers soient absents; mais ils sont fréquemment implicites et restent souvent à établir sur la base d'un calcul d'inférence issu de l'appréciation des contenus sémantiques et des topoï de la doxa. (Boix 77-78)

Comme nous avons eu l'occasion de le constater, cette variation diachronique ne semble pas opérer dans le cas précis des proverbes, qui semblent au contraire conserver ces liens logiques pour expliciter le rapport sémantique qui doit être établi entre les membres du proverbe. Ce que nous constatons dans 
le cadre des énoncés sentencieux s'approche davantage des logiques inhérentes aux langues. Dans le cadre des proverbes, s'appliquent ainsi des constats relevés plus globalement dans toute communication:

D'une manière générale, le rapport logique de cause à conséquence est marqué en espagnol par une simple parataxe asyndétique, qui laisse le soin à l'interlocuteur de saisir le rapport entretenu par les deux énoncés en se reportant à leurs contenus propositionnels. Mais dès lors que le rapport deviendra coûteux, ou difficile à accepter par la personne même qui l'établit, le locuteur devra alors le marquer explicitement. (Fretel 2007, 361-62)

La présence dans les proverbes relevés d'un marquage explicite est de nature à nous interpeller: quelles sont, dans ces conditions, les relations qui doivent être marquées par une conjonction?

L'apparition de la relation causale ou consécutive véhiculée par pues/car peut être rapidement traitée: ces connecteurs indiquant la prémisse ne pouvant pas être déplacés. Leur présence s'inscrit à notre sens dans un renforcement du lien causal ou consécutif qui ne contredit pas la nature implicative de l'énoncé en question. Dans No pidas al olmo la pera, pues no la lleva, ou dans "Cœur de femme trompe le monde, car en lui malice abonde", les associations d'idées (qui contreviennent aux lois du monde et la doxa, en quelque sorte) sont peut-être à l'origine du renforcement du lien par l'ajout d'une conjonction, mais celles-ci ne modifient pas la lecture implicative de l'énoncé.

Les proverbes qui se construisent sur une relation adversative doivent en revanche attirer plus particulièrement notre attention. Construits en français grâce à la conjonction mais, ils acceptent en espagnol deux conjonctions qui à leur tour nuancent le contenu des propositions mises en présence.

(9a) La traición aplace, mas no el que la hace

(9b) No te digo que te vayas, pero ahí tienes la puerta

Pero (ou mas) introduit une restriction après un verbe affirmatif (9a) ou négatif (9b). Ces deux conjonctions (synonymes) disqualifient d'un point de vue argumentatif l'affirmation première sans l'annuler. Dans nos deux exemples, la première proposition oriente vers une certaine conclusion, non formulée: la trahison est plaisante, celui qui trahit l'est aussi; je ne te demande pas de partir (donc reste). Des propositions qui vont être elles-mêmes nuancées par l'ajout de la proposition introduite par pero, car celle-ci exprime un argument plus fort que 
la première et impose la conclusion opposée, à savoir pour nos exemples: le traître est déplaisant, tu ferais mieux de partir.

(10) No es por el huevo, sino por el fuero

Sino s'emploie dans une phrase négative (condition nécessaire mais non suffisante) pour introduire une opposition entre deux arguments incompatibles. Sino remplace ainsi l'affirmation première en l'annulant (10). Il permet de reformuler positivement et de définir ce que la première proposition présente négativement. Dans les propositions adversatives avec sino, le premier argument est balayé: el huevo, et laisse place au deuxième argument, le seul valable: el fuero.

Ces conjonctions sont nécessaires dans les deux cas, car le mécanisme de la langue veut que lorsque deux événements sont successifs, sauf en cas d'indication contraire, la lecture logique soit que le second est la conséquence du premier.

(11) Pierre est venu, Marc est parti.

L'arrivée de Pierre a été un argument, a impliqué le départ de Marc. Le jeu de l'implication se trouve déjà dans les règles de la langue. Et par conséquent dans les proverbes. En dehors de toute spécification, par exemple, la présence d'une conjonction de coordination venant spécifier le type de relation qui lie les deux membres de l'énoncé, la lecture à faire sera toujours de nature implicative. En revanche la présence d'une conjonction agit comme un contre ordre: elle est en charge de dire le lien qui s'instaure entre les deux membres.

\section{CONCLUSION}

Le corpus de proverbes sélectionné et l'outil diachronique précisent encore les propriétés linguistiques du proverbe et permettent d'en préciser la définition. Posé comme un élément permettant de mieux appréhender l'énoncé sentencieux dans les domaines de la syntaxe et de la sémantique, le concept de l'implication qui lie les deux membres d'un proverbe mérite d'être encore approfondi, notamment car il ne peut s'appliquer à l'ensemble des énoncés sentencieux. Quant à la diachronie, elle permet de vérifier la stabilité de la structure syntaxique de ces énoncés, et de poser l'hypothèse, au vu du maintien des conjonctions au sein des énoncés parémiques, de leur nécessité sémantique. 


\section{OUVRAGES CITÉS}

Achard-Bayle, Guy y Bertille Schneider. "Les énoncés parémiques hypo- et paratactiques: des constructions syntaxiques aux interprétations sémantiques". La Parataxe: Structures, marquages et exploitations discursives. Vol. 2. Eds. Marie-Josée Béguelin, Mathieu Avanzi et Gilles Corminboeuf. Sciences pour la communication 92. Bern/etc.: Peter Lang, 2010. 95-120. Anscombre, Jean-Claude. "Proverbes et formes proverbiales: valeur évidentielle et argumentative". Langue française 102 (1994): 95-107.

Anscombre, Jean-Claude. "Semántica y léxico: topoï, estereotipos y frases genéricas”. Revista Española de Lingüística 25.2 (1996): 297-310.

Anscombre, Jean-Claude. "Reflexiones críticas sobre la naturaleza y el funcionamiento de las paremias". Paremia 6 (1997): 43-54.

Anscombre, Jean-Claude. "Paroles proverbiales et structures métriques". Langages 139 (2000): 6-26.

Anscombre, Jean-Claude. "Pour une théorie linguistique du phénomène parémique". La Parole exemplaire: Introduction à une étude linguistique des proverbes. Eds. Jean-Claude Anscombre, Bernard Darbord et Alexandra Oddo. Paris: Armand Colin, 2012a. 21-39.

Anscombre, Jean-Claude. "Matrices rythmiques et parémies". La Parole exemplaire: Introduction à une étude linguistique des proverbes. Eds. Jean-Claude Anscombre, Bernard Darbord et Alexandra Oddo. Paris: Armand Colin, 2012b. 147-58.

Boix, Christian. "Du minimalisme argumentatif dans le discours contemporain”. Argumentation, manipulation, persuasion (Actes du colloque organisé par le Laboratoire de Recherches en Langues et Littératures Romanes, Études Basques, Espace Caraïbe de l'Université de Pau et des Pays de l'Adour, du 31 mars au 2 avril 2005). Ed. Christian Boix. Paris: L'Harmattan, 2007. 73-86.

Caro y Cejudo, Gerónimo Martín. Refranes y Modos de bablar Castellanos, con los Latinos que les corresponden; y la glosa y explicacion de los que tienen necesidad de ella. 1675. Madrid: Imprenta real, 1792.

Correas, Gonzalo. Vocabulario de refranes y frases proverbiales. 1627. Ed. Louis Combet. Bordeaux: Institut d'Études ibériques et ibéro-Américaines de l'Université de Bordeaux, 1967.

Doval, Gregorio. Refranero temático español. Madrid: Ediciones del Prado, 1997. Ducrot, Oswald y Jean-Marie Schaeffer. Nouveau Dictionnaire encyclopédique des sciences du langage. Paris: Éditions du Seuil, 1995. 
Esbozo: Real Academia Española. Esbozo de una nueva gramática de la lengua española. Madrid: Espasa Calpe, 1973.

Fretel, Hélène. "Le locuteur: un stratège manipulé". Argumentation, manipulation, persuasion (Actes du colloque organisé par le Laboratoire de Recherches en Langues et Littératures Romanes, Études Basques, Espace Caraïbe de l'Université de Pau et des Pays de l'Adour, du 31 mars au 2 avril 2005). Ed. Christian Boix. Paris: L'Harmattan, 2007. 368-69.

García de Castro, Diego. Seniloquium. ca. 1480. Eds. Fernando Cantalapiedra Erostarbe y Juan Moreno Uclés. Valencia: Publicacions de la Universitat de València, 2006.

Gómez-Jordana, Sonia. Le Proverbe: vers une définition linguistique. Étude sémantique des proverbes français et espagnols contemporains. Paris: L'Harmat$\tan , 2012 \mathrm{a}$.

Gómez-Jordana, Sonia. "Les moules proverbiaux en français contemporain". La Parole exemplaire: Introduction à une étude linguistique des proverbes. Eds. Jean-Claude Anscombre, Bernard Darbord et Alexandra Oddo. Paris: Armand Colin, 2012b. 114-32.

Grevisse, Maurice y André Goosse. Le bon usage. 14.a ed. Bruxelles: De Boeck Duculot, 2007.

Junceda, Luis. Diccionario de refranes, dichos y proverbios. Madrid: Espasa-Calpe, 1998.

Kleiber, Georges. "Sur la définition du proverbe". Recherches Germaniques 2 (1989): 233-52.

Kleiber, Georges. "Sur le sens des proverbes". Langages 139 (2000): 39-58.

Le Roux de Lincy, Antoine. Le Livre des proverbes français. Paris: Adolphe Delahays, 1859.

"Libro de adverbios". Glosarios latino-españoles de la Edad Media. Ca. 1400. Ed. Américo Castro. Madrid: Biblioteca de Filología Hispánica 3. 19361991.

Marqués de Santillana, Íñigo López de Mendoza. Refranes que dizen las viejas tras el fuego. 1454. Ed. Hugo O. Bizarri. Barcelona: Reichenberger, 1995.

Morawski, Joseph. Proverbes antérieurs au XVème siècle. Paris: Champion, 1925.

Nicot, Jean. Thrésor de la langue françoyse, tant ancienne que moderne. Paris: chez David Douceur, 1606.

Núñez, Hernán. Refranes y proverbios en romance. 1555. Eds. Louis Combet, Julia Sevilla Muñoz, Germán Conde Tarrío et Josep Guia. Madrid: Guillermo Blázquez, 2001. 
Oddo, Alexandra. "Évolution du Refranero castillan: la question des proverbes tronqués". Crisol 14 (2011): 97-113.

Oddo, Alexandra. "Phénomènes de troncature". La Parole exemplaire: Introduction à une étude linguistique des proverbes. Eds. Jean-Claude Anscombre, Bernard Darbord et Alexandra Oddo. Paris: Armand Colin, 2012. 133-46.

Oddo, Alexandra. Vers un refranero diachronique: analyse linguistique de l'évolution linguistique des proverbes espagnols depuis le Moyen Âge. Limoges: Lambert Lucas, 2013.

Pottier, Bernard. Sémantique générale. 3.a ed. Paris: PUF, 2011.

Riegel, Martin. “«Qui dort dîne» ou le pivot implicatif dans les énoncés parémiques”. Travaux de linguistique et de littérature 24.1 (1987): 85-99.

Sbarbi, José María. El Libro de los Refranes: colección alfabética de refranes castellanos explicados con la mayor concisión y claridad. Madrid: Librería de D. Leon Pablo Villaverde, 1872.

Sbarbi, José María. Diccionario de refranes, adagios, proverbios, modismos, locuciones y frases proverbiales de la lengua española. Ed. Manuel José García. Madrid: Librería de los Sucesores de Hernando, 1922.

Vallés, Pedro. Libro de refranes y sentencias. 1549. Eds. Jesús Cantera y Julia Sevilla. Madrid: Guillermo Blázquez, 2003. 